\title{
Evaluation of Lingual Arch form among Angle Class I, Class Iiand Class III Malocclusions in Chinese Ortho- dontic Patients
}

\author{
Hong Chen ${ }^{1}$, Shaohua Song ${ }^{2}$, Nuo Zhou ${ }^{2 *}$
}

\begin{abstract}
${ }^{1}$ Department of Stomatology, the Ninth Affiliated Hospital of Guangxi Medical University, Beihai, Guangxi, China, 69 He Ping Road, Beiahi, Guangxi 536000, China

${ }^{2}$ Guangxi Medical University College of Stomatology, Nanning, Guangxi, China, No.22 Shuang-Yong Road, Qing-Xue District, Nanning, Guangxi 530021, China
\end{abstract}

*Corresponding author: Nuo Zhou, Guangxi Medical University College of Stomatology, Nanning, Guangxi, China, No.22 Shuang-Yong Road, Qing-Xue District, Nanning, Guangxi 530021. China, Tel: 86-771-5352512; Fax: 86-771-5356491; E-mail: nzhou.gxmu@gmail.com

\begin{abstract}
:
Introduction: The aim of this study was to analyze and compare the dental lingual arch widths and lengths, alveolar arch within patients with Angle's Class I (ACI), Class IIdivision1 (ACII) and Class III (ACIII) malocclusions in Chinese patients.

Methods: This study was performed using measurements on ninety dental casts with ACI (age: $22.2 \pm 3.6$ ), ACII (age: $23.0 \pm 3.8$ ), and ACIII (age: $22.0 \pm 2.8$ ) malocclusion subjects. Two-way ANOVA post-hoc and nonparametric tests were applied for comparisons in groups and genders.

Results: Maxillary canineand premolar alveolar widths and all maxillary lingual lengths were significantly narrower in ACIII $(\mathrm{p}<0.05)$. The mandibular intercanine andintermolar widths were wider and the maxillary intermolar width measurement was larger in ACIII $(\mathrm{p}<0.05)$. Male's palate height in ACIII was greater than ACII $(p<0.05)$. Gender differences were also significant in these measurements.

Conclusions: Our data showed undergrowth of the maxilla and overgrowth of the mandible and incisor inclined lingual in ACIII; mandibular growth retrusion and maxillary incisor inclined in ACII. Alveolar width was greater in males than females.
\end{abstract}

Keywords: Lingual arch form; Alveolar arch width; Lingual arch width; Lingual arch length; Malocclusions

\section{Introduction}

The dental arch, fundamental principle in orthodontic planning and therapy, is an important element in orthodontics ${ }^{[1]}$. Therefore, correct identification of a patient's arch form is a crucial parameter in achieving a stable, functional and esthetic orthodontic treatment result, since failure to preserve the arch form might increase the probability of relapse ${ }^{[2]}$.

Lingual orthodontics was developed by the end of the 70's with the bonding of conventional appliances on the lingual surface of $\operatorname{teeth}^{[3]}$. The first study describing brackets and lingual arch shape was published in 40 years ago ${ }^{[4]}$. Since then, there are many confounding factors on measuring intercanine distances which hinder clinicians from determining the size of mushroom-shaped lingual arches. To simplify this technique, researchers introduced a new anatomic arch form technique in Lingual Orthodontics ${ }^{[5]}$ and proposed the positioning of thicker bracket bases to allow the use of arch wires without offsets ${ }^{[6]}$. They obtained arch form by the different method, some used the 3D digital imaging to obtain a three-dimensional structural model, and some adopted the teeth landmarks such as the tongue side of the center or crown cusp positioned, while others applied geometric curves to describe the dental $\operatorname{arch}^{[7-10]}$.

Although the average arch form of a normal occlusion sample was provided, a classification of the lingual arch forms per shape or size has not been attempted. Most of the
Received date: August 16, 2018 Accepted date: July 20, 2018

Published date: July 24, 2018

Citation: Zhou, N., et al. Evaluation of Lingual Arch form among Angle Class I, Class Iiand Class III Malocclusions in Chinese Orthodontic Patients. (2018) J Dent Oral Care 4(2): 30- 37 .

Copyright: (C) 2018 Zhou, N. This is an Open access article distributed under the terms of Creative Commons Attribution 4.0 International License. 
studies in the literature compare dental arch widths of Skeletal Class II (SC II) patients with the normal occlusion samples ${ }^{[7,11-13]}$. Some of them indicate absolute arch widths of children with malocclusion did not differ appreciably from those with normal occlusion $^{[7,12]}$. However, in other studies, statistically significant differences were determined in dental and alveolar width measurements of SC II patients ${ }^{[13]}$.

Our review of the literature revealed few studies evaluated group of gender differences of lingual arch form among Angle's Class I (AC I), Class II (AC II) and Class III (AC III) malocclusions in Chinese orthodontic patients. Therefore, the aim of this study was to compare difference in maxillary and mandibular dental arch and alveolar width dimensions among these three lingual Angle Classes, as well as the gender differences within each arch form clarified for Angle classification. This research might be interesting and can provide reference for our clinicians in the choice of orthodontic lingual wires of AC I, II and III patients.

\section{Materials and Methods}

The sample consisted of 90 young adults with AC I, AC II division1, and AC III malocclusion (45 males and 45 females) randomly selected from a database of 7,521 Chinese orthodontic patients. The distribution of age in different groups for all subjects is shown in Table 1.

Table 1: The Distribution of age in different malocclusion groups

\begin{tabular}{|l|l|l|l|l|l|}
\hline & Sample & Mean Age(y) & $\operatorname{SD}(\mathrm{y})$ & $\operatorname{Max}(\mathrm{y})$ & $\operatorname{Min}(\mathrm{y})$ \\
\hline ACI Female) & 15 & 22.6 & 3.31 & 30 & 19 \\
\hline ACI (Male) & 15 & 21.73 & 3.90 & 30 & 17 \\
\hline ACII (Female) & 15 & 22.87 & 3.20 & 29 & 17 \\
\hline ACII (Male) & 15 & 23.13 & 4.39 & 30 & 17 \\
\hline ACIII (Female) & 15 & 22.00 & 2.78 & 29 & 19 \\
\hline ACIII (Male) & 15 & 21.93 & 2.76 & 28 & 19 \\
\hline
\end{tabular}

ACI: Angle Class I malocclusion; ACII: Angle Class II malocclusion; ACIII: Angle Class III malocclusion; SD: standard deviation; Min: minimum; and Max: maximum.

The inclusion criteria were as follows:

- AC I: bilateral Class I molar and canine relationships incentric occlusion, $0^{\circ}<\mathrm{ANB}<5^{\circ}$; over bite and over jet $<4 \mathrm{~mm}$;

- AC II: bilateral Class II molar and canine relationship incentric occlusion, $5^{\circ}<\mathrm{ANB}$, protrusive maxillary incisors; convex soft tissue profile, excessive over jet $>3 \mathrm{~mm}$, absence of posterior cross bite;

- AC III: bilateral Class III molar and canine relationships, ANB

$<0^{\circ}$, concave soft tissue profile, negative over jet $>1 \mathrm{~mm}$; negative over bite $>1 \mathrm{~mm}$;

The exclusion criteria were as follows:

- Minor tooth size - arch length discrepancy $<2 \mathrm{~mm}$ crowding,

$<2$ mm spacing;

- Flat or slight curve of Speed $<2 \mathrm{~mm}$; absence of dental midline deviation;

- Permanent dentition with normal tooth size and shape, except third molars;

- No open bite and no teeth of severe out of dental arch and torsion;
Ninety maxillary and mandibular dental and alveolar widths, arch lengths measurements were performed on the dental casts of each subject. These measurements were recorded from each subject's dental casts by one examiner, using aVernier caliper (Shanghai Tool Works Inc., China) and recording the data to the nearest $0.02 \mathrm{~mm}$. These dental and alveolar arch width measurements are shown as Figure 1 and Table 2. Three weeks after the first measurements, 20 dental casts were selected randomly and re-measured. The difference between the first and second measurements was insignificant. Intra-examiner reliability test was performed with the Cronbach's Alpha is 0.95 .

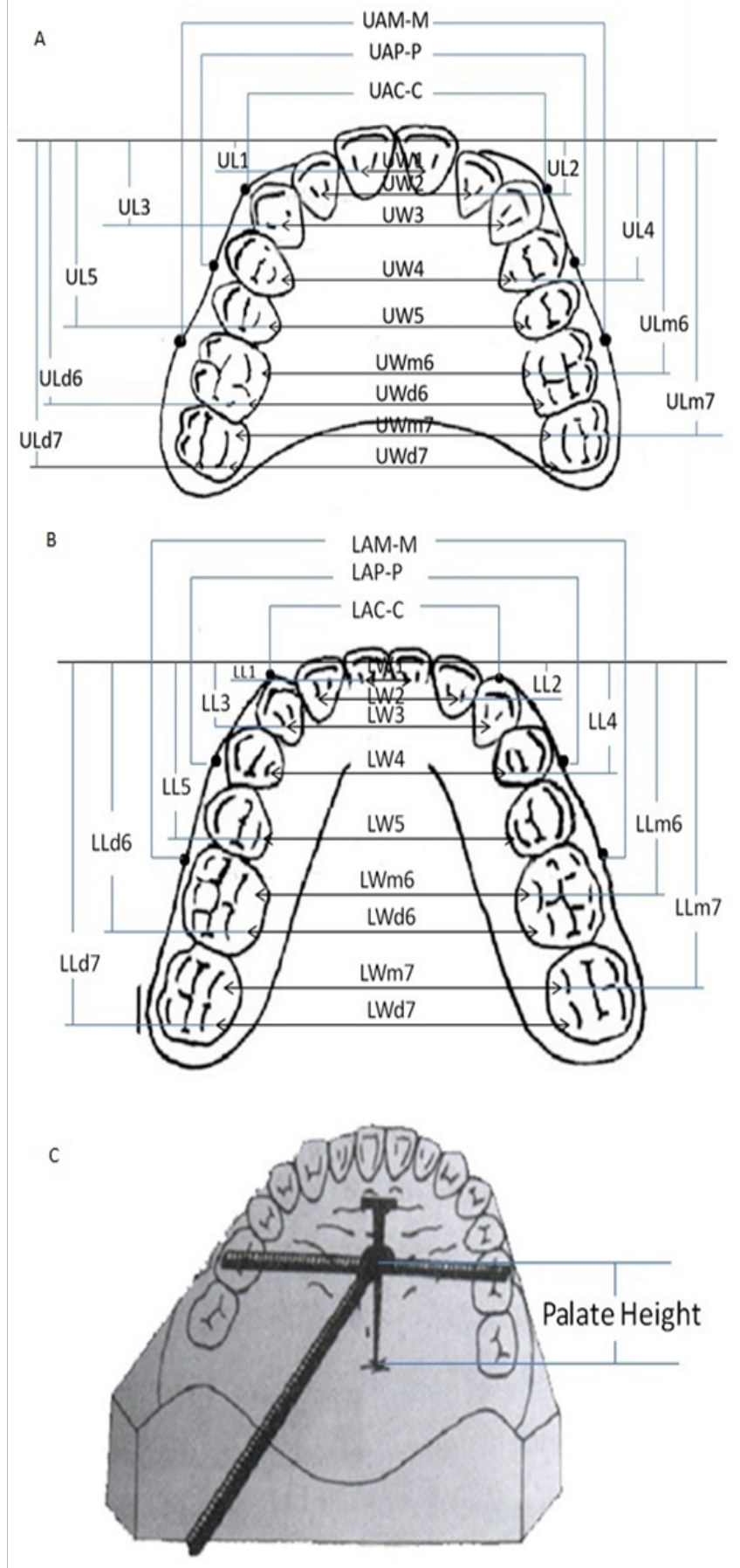

Figure 1: Illustration of measurement parameters in Table 2: A. Maxillary dental and alveolar width; B. Mandibular dental and alveolar width; and C. Palate height. 
Citation: Zhou, N., et al. Evaluation of Lingual Arch form among Angle Class I, Class Iiand Class III Malocclusions in Chinese Orthodontic Patients. (2018) J Dent Oral Care 4(2): 30- 37.

Table 2: Maxillary and mandibular dental and alveolar width measurement parameters.

\begin{tabular}{|c|c|}
\hline $\mathrm{UW}_{1}$ & $\begin{array}{l}\text { The distance between the center of the lingual clinical } \\
\text { crown of the maxillary right and left central incisors }\end{array}$ \\
\hline $\mathrm{UW}_{2}$ & $\begin{array}{l}\text { The distance between the center of the lingual clinical } \\
\text { crown of the maxillary right and left lateral incisors }\end{array}$ \\
\hline $\mathrm{UW}_{3}$ & $\begin{array}{l}\text { The distance between the center of the lingual clinical } \\
\text { crown of the maxillary right and left canines }\end{array}$ \\
\hline $\mathrm{UW}_{4}$ & $\begin{array}{l}\text { The distance between the center of the lingual clinical } \\
\text { crown of the maxillary right and left first premolars }\end{array}$ \\
\hline $\mathrm{UW}_{5}$ & $\begin{array}{l}\text { The distance between the center of the lingual clinical } \\
\text { crown of the maxillary right and left second premolars }\end{array}$ \\
\hline $\mathrm{UWm}_{6}$ & $\begin{array}{l}\text { The distance between the center of the mesial-lingual sur- } \\
\text { face of the maxillary right and left first molars }\end{array}$ \\
\hline $\mathrm{UWd}_{6}$ & $\begin{array}{l}\text { The distance between the center of the distal-lingual sur- } \\
\text { face of the maxillary right and left first molars }\end{array}$ \\
\hline $\mathrm{UWm}_{7}$ & $\begin{array}{l}\text { The distance between the center of the mesial-lingual sur- } \\
\text { face of the maxillary right and left second molars }\end{array}$ \\
\hline $\mathrm{UWd}_{7}$ & $\begin{array}{l}\text { The distance between the center of the distal-lingual sur- } \\
\text { face of the maxillary right and left second molars }\end{array}$ \\
\hline $\mathrm{LW}_{1}$ & The projection of UW1 point in mandible \\
\hline $\mathrm{LW}_{2}$ & The projection of UW2 point in mandible \\
\hline $\mathrm{LW}_{3}$ & The projection of UW3 point in mandible \\
\hline $\mathrm{LW}_{4}$ & The projection of UW4 point in mandible \\
\hline $\mathrm{LW}_{5}$ & The projection of UW5 point in mandible \\
\hline $\mathrm{LWm}_{6}$ & The projection of UWm6 point in mandible \\
\hline $\mathrm{LWd}_{6}$ & The projection of UWd6 point in mandible \\
\hline $\mathrm{LWm}_{7}$ & The projection of UWm7 point in mandible \\
\hline $\mathrm{LWd}_{7}$ & The projection of UWd7 point in mandible \\
\hline $\mathrm{UL}_{1}$ & $\begin{array}{l}\text { The shortest distance from a line connecting the center } \\
\text { of the lingual clinical crown of the maxillary right and } \\
\text { left central incisors to the proximal contact point of the } \\
\text { lingual maxillary central incisors }\end{array}$ \\
\hline
\end{tabular}

\begin{tabular}{|l|l|}
\hline $\mathrm{UL}_{2}$ & The shortest distance from a line connecting the center of
\end{tabular} the lingual clinical crown of the maxillary right and left lateral incisors to the proximal contact point of the lingual maxillary central incisors

\begin{tabular}{|l|l}
$\mathrm{UL}_{3}$ & The shortest distance from a line connecting the center
\end{tabular} of the lingual clinical crown of the maxillary right and left canines to the proximal contact point of the lingual maxillary central incisors

\begin{tabular}{l|l}
$\mathrm{UL}_{4}$ & The shortest distance from a line connecting the center of
\end{tabular} the lingual clinical crown of the maxillary right and left first premolars to the proximal contact point of the lingual maxillary central incisors

\begin{tabular}{l|l}
$\mathrm{UL}_{5}$ & The shortest distance from a line connecting the center
\end{tabular} of the lingual clinical crown of the maxillary right and left second premolars to the proximal contact point of the lingual maxillary central incisors

\begin{tabular}{l|l}
$\mathrm{ULm}_{6}$ & The shortest distance from a line connecting the center of
\end{tabular} the mesial-lingual surface of the maxillary right and left first molars to the proximal contact point of the lingual maxillary central incisors

\begin{tabular}{l|l} 
ULd $_{6}$ & The shortest distance from a line connecting the center of
\end{tabular} the distal-lingual surface of the maxillary right and left first molars to the proximal contact point of the lingual maxillary central incisors

\begin{tabular}{|c|c|}
\hline $\mathrm{ULm}_{7}$ & $\begin{array}{l}\text { The shortest distance from a line connecting the center of } \\
\text { the mesial-lingual surface of the maxillary right and left } \\
\text { second molars to the proximal contact point of the lingual } \\
\text { maxillary central incisors }\end{array}$ \\
\hline $\mathrm{ULd}_{7}$ & $\begin{array}{l}\text { The shortest distance from a line connecting the center of } \\
\text { the distal-lingual surface of the maxillary right and left } \\
\text { second molars to the proximal contact point of the lingual } \\
\text { maxillary central incisors }\end{array}$ \\
\hline $\mathrm{LL}_{1}$ & The projection of UL1 point in mandible \\
\hline $\mathrm{LL}_{2}$ & The projection of UL2 point in mandible \\
\hline $\mathrm{LL}_{3}$ & The projection of UL3 point in mandible \\
\hline $\mathrm{LL}_{4}$ & The projection of UL4 point in mandible \\
\hline $\mathrm{LL}_{5}$ & The projection of UL5 point in mandible \\
\hline $\mathrm{LLm}_{6}$ & The projection of ULm6 point in mandible \\
\hline $\operatorname{LLd}_{6}$ & The projection of ULd6 point in mandible \\
\hline $\mathrm{LLm}_{7}$ & The projection of ULm7 point in mandible \\
\hline $\operatorname{LLd}_{7}$ & The projection of ULd7 point in mandible \\
\hline UAC-C & $\begin{array}{l}\text { The distance between two points at the mucogingival } \\
\text { junctions above the cusp tips of the maxillary right and } \\
\text { left canines. }\end{array}$ \\
\hline LAC-C & The projection of UAC-C point in mandible \\
\hline UAP-P & $\begin{array}{l}\text { The distance between two points at the mucogingival } \\
\text { junctions above the inter-dental contact point of the max- } \\
\text { illary first and second premolars. }\end{array}$ \\
\hline LAP-P & The projection of UAP-P point in mandible \\
\hline UAM-M & $\begin{array}{l}\text { The distance between two points at the mucogingival } \\
\text { junctions above the mesiobuccal cusp tips of the maxil- } \\
\text { lary first molars }\end{array}$ \\
\hline LAM-M & The projection of UAM-M point in mandible \\
\hline $\begin{array}{l}\text { P a la te } \\
\text { Height }\end{array}$ & $\begin{array}{l}\text { The shortest distance from palatal dome to the ruler edge } \\
\text { distance when the ruler placed the occlusal surface of } \\
\text { maxillary first molars and the edge of rule through the } \\
\text { central fossa, }\end{array}$ \\
\hline
\end{tabular}

The data that show the normal distribution used twoway ANOVA and post-hoc tests to apply for comparison of the groups. And syntax was applied for comparison between genders in the group. The data that show the non-normal distribution used nonparametric tests: Wilcoxon and kruskal-Wallis test. All statistical analyses were performed using the SPSS software package (SPSS Inc, Chicago, Ill).

\section{Results}

Pertwo-way ANOVA and post-hoc tests, the statistically significant group differences were observed in $\mathrm{UL}_{1}, \mathrm{UL}_{3}, \mathrm{UL}_{5}, \mathrm{ULm}_{6}$, $\mathrm{ULd}_{6}, \mathrm{ULm}_{7}, \mathrm{ULd}_{7}, \mathrm{LL}_{1}$; and significant gender differences were showed in $\mathrm{UW}_{5}, \mathrm{UL}_{3}, \mathrm{UL}_{5}, \mathrm{ULm}_{6}, \mathrm{ULd}_{6}, \mathrm{ULm}_{7}, \mathrm{ULd}_{7}, \mathrm{LL}_{3}, \mathrm{LL}_{4}$, LLm $_{6}, \mathrm{LLd}_{6}, \mathrm{LLm}_{7}$, UAP-P, UAM-M and Palate Height; the interaction of group and genders were in $\mathrm{UW}_{2}$ and $\mathrm{ULm}_{6}$, see Table 3.

\section{Gender differences on Angle's Class I, II and III malocclu- sions}

Descriptive statistics and statistical comparisons of dental lingual arch widths and lengths and alveolar width measurements for dental casts ACI, II and III are shown in Table 3. 
Table 3: Descriptive statistics and gender differences of maxillary and mandibular lingual arch widths \& lengths and alveolar width measurements on ACI, ACII and ACIII malocclusions.

\begin{tabular}{|c|c|c|c|c|c|c|}
\hline Parameters & ACI & & ACII & & ACIII & \\
\hline & Female (mm) & Male (mm) & Female (mm) & Male (mm) & Female (mm) & Male (mm) \\
\hline $\mathrm{UW}_{1}$ & $8.45 \pm 0.58$ & $8.85 \pm 1.13$ & $8.54 \pm 0.77$ & $8.95 \pm 0.57$ & $8.20 \pm 0.58$ & $8.25 \pm 0.84$ \\
\hline $\mathrm{UW}_{2}{ }^{\#}$ & $21.46 \pm 0.85$ & $21.17 \pm 2.04$ & $20.34 \pm 1.73$ & $22.1 \pm 1.13^{\mathrm{bb}}$ & $20.65 \pm 1.28$ & $20.93 \pm 1.06$ \\
\hline $\mathrm{UW}_{3}$ & $31.20 \pm 1.91$ & $31.19 \pm 2.61$ & $30.14 \pm 1.84$ & $31.63 \pm 1.45^{b}$ & $31.74 \pm 1.70$ & $30.81 \pm 4.37$ \\
\hline $\mathrm{UW}_{4}$ & $30.01 \pm 2.33$ & $30.81 \pm 2.76$ & $28.88 \pm 2.39$ & $30.89 \pm 1.63^{\mathrm{bb}}$ & $30.44 \pm 2.06$ & $31.07 \pm 2.23$ \\
\hline $\mathrm{UW}_{5}{ }^{*}$ & $34.56 \pm 2.70$ & $36.16 \pm 3.96$ & $32.83 \pm 2.09$ & $34.88 \pm 1.31^{\mathrm{b}}$ & $34.84 \pm 2.53$ & $35.49 \pm 2.67$ \\
\hline $\mathrm{UWm}_{6}$ & $36.95 \pm 2.73$ & $39.31 \pm 3.67$ & $35.55 \pm 2.47$ & $37.27 \pm 1.63$ & $38.26 \pm 1.70$ & $38.86 \pm 2.96$ \\
\hline $\mathrm{UWd}_{6}$ & $39.24 \pm 2.83$ & $41.43 \pm 3.97$ & $37.49 \pm 2.55$ & $39.94 \pm 2.03^{b}$ & $40.35 \pm 1.81$ & $41.55 \pm 3.21$ \\
\hline $\mathrm{UWm}_{7}$ & $41.94 \pm 3.02$ & $44.89 \pm 4.97$ & $40.52 \pm 2.26$ & $43.01 \pm 2.19^{\mathrm{bb}}$ & $43.18 \pm 1.76$ & $45.05 \pm 3.45$ \\
\hline $\mathrm{UWd}_{7}$ & $44.96 \pm 3.45$ & $47.78 \pm 5.35$ & $43.28 \pm 2.43$ & $45.15 \pm 2.44^{b}$ & $44.67 \pm 2.01$ & $46.93 \pm 3.35^{c}$ \\
\hline $\mathrm{LW}_{1}$ & $5.51 \pm 0.21$ & $5.47 \pm 0.40$ & $5.27 \pm 0.29$ & $5.50 \pm 0.45$ & $5.40 \pm 0.37$ & $5.39 \pm 0.33$ \\
\hline $\mathrm{LW}_{2}$ & $15.56 \pm 1.92$ & $15.42 \pm 1.71$ & $13.9 \pm 1.58$ & $14.88 \pm 0.92$ & $15.37 \pm 0.80$ & $15.26 \pm 0.77$ \\
\hline $\mathrm{LW}_{3}$ & $23.41 \pm 2.31$ & $24.41 \pm 1.86$ & $23.07 \pm 1.62$ & $24.91 \pm 1.36^{\mathrm{bb}}$ & $25.29 \pm 1.32$ & $25.25 \pm 1.97$ \\
\hline $\mathrm{LW}_{4}$ & $27.04 \pm 2.63$ & $27.67 \pm 3.20$ & $26.91 \pm 1.81$ & $27.84 \pm 1.3$ & $28.09 \pm 1.78$ & $28.03 \pm 2.42$ \\
\hline $\mathrm{LW}_{5}$ & $31.01 \pm 2.63$ & $31.46 \pm 3.51$ & $30.26 \pm 2.38$ & $31.55 \pm 1.63$ & $31.71 \pm 2.37$ & $32.46 \pm 2.79$ \\
\hline $\mathrm{LWm}_{6}$ & $32.91 \pm 2.83$ & $34.02 \pm 3.8$ & $32.35 \pm 2.18$ & $33.95 \pm 1.72$ & $34.66 \pm 1.79$ & $35.53 \pm 2.89$ \\
\hline $\mathrm{LWd}_{6}$ & $34.96 \pm 2.76$ & $36.90 \pm 3.81$ & $34.74 \pm 1.91$ & $36.86 \pm 1.96 \mathrm{~b}$ & $36.93 \pm 1.95$ & $38.49 \pm 3.38$ \\
\hline $\mathrm{LWm}_{7}$ & $38.7 \pm 2.94$ & $40.30 \pm 4.01$ & $37.98 \pm 2.15$ & $40.10 \pm 2.00^{\mathrm{b}}$ & $39.83 \pm 1.54$ & $41.4 \pm 3.06$ \\
\hline $\mathrm{LWd}_{7}$ & $40.43 \pm 2.90$ & $42.53 \pm 3.85$ & $39.76 \pm 1.85$ & $42.00 \pm 2.14^{\mathrm{bb}}$ & $41.78 \pm 1.67$ & $43.93 \pm 3.30^{c}$ \\
\hline $\mathrm{UL}_{1} \& \&$ & $0.57 \pm 0.12$ & $0.64 \pm 0.09$ & $0.68 \pm 0.08$ & $0.70 \pm 0.07$ & $0.67 \pm 0.08$ & $0.70 \pm 0.09$ \\
\hline $\mathrm{UL}_{2}$ & $3.06 \pm 0.80$ & $3.56 \pm 0.37^{\mathrm{a}}$ & $3.23 \pm 0.52$ & $4.15 \pm 0.55^{\mathrm{bb}}$ & $3.15 \pm 0.81$ & $3.56 \pm 1.33$ \\
\hline $\mathrm{UL}_{3}^{* *}, \& \&$ & $8.32 \pm 1.36$ & $8.73 \pm 1.23$ & $8.16 \pm 0.77$ & $9.56 \pm 1.33^{\mathrm{bb}}$ & $6.04 \pm 1.02$ & $7.58 \pm 1.88^{\mathrm{cc}}$ \\
\hline $\mathrm{UL}_{4}$ & $16.85 \pm 1.45$ & $17.81 \pm 1.61$ & $17.21 \pm 1.38$ & $18.33 \pm 1.15^{\mathrm{b}}$ & $14.27 \pm 1.37$ & $16.58 \pm 2.34^{\mathrm{cc}}$ \\
\hline $\mathrm{UL}_{5}^{* *, \& \&}$ & $23.71 \pm 1.61$ & $24.49 \pm 2.05$ & $23.36 \pm 1.53$ & $25.72 \pm 1.46^{\mathrm{bb}}$ & $21.15 \pm 1.45$ & $23.68 \pm 2.4^{\mathrm{cc}}$ \\
\hline $\mathrm{ULm}_{6}^{* *, \& \&, \#}$ & $30.67 \pm 2.18$ & $31.38 \pm 2.21$ & $30.37 \pm 1.57$ & $32.59 \pm 1.01^{\mathrm{bb}}$ & $27.67 \pm 1.17$ & $30.77 \pm 2.53^{\mathrm{cc}}$ \\
\hline $\mathrm{ULd}_{6}^{* *, \& \&}$ & $34.98 \pm 2.18$ & $35.84 \pm 2.40$ & $34.97 \pm 1.48$ & $37.41 \pm 1.21^{\mathrm{bb}}$ & $32.18 \pm 1.38$ & $35.03 \pm 2.65^{\mathrm{cc}}$ \\
\hline $\mathrm{ULm}_{7}^{* *}, \& \&$ & $41.00 \pm 2.30$ & $41.78 \pm 2.81$ & $40.41 \pm 1.9$ & $43.31 \pm 1.09^{\mathrm{bb}}$ & $37.91 \pm 1.40$ & $40.73 \pm 2.62^{\mathrm{cc}}$ \\
\hline $\mathrm{ULd}_{7}^{* *}, \& \&$ & $44.22 \pm 2.12$ & $45.52 \pm 2.64$ & $44.26 \pm 2.00$ & $46.80 \pm 1.12^{\mathrm{bb}}$ & $41.57 \pm 1.49$ & $44.26 \pm 2.68^{\mathrm{cc}}$ \\
\hline $\mathrm{LL}_{1}^{\& \&}$ & $0.44 \pm 0.10$ & $0.46 \pm 0.09$ & $0.50 \pm 0.06$ & $0.46 \pm 0.08$ & $0.39 \pm 0.05$ & $0.43 \pm 0.06$ \\
\hline $\mathrm{LL}_{2}$ & $1.80 \pm 0.64$ & $2.04 \pm 0.25$ & $1.88 \pm 0.21$ & $2.14 \pm 0.16^{\mathrm{bb}}$ & $1.59 \pm 0.19$ & $1.79 \pm 0.20^{\mathrm{cc}}$ \\
\hline $\mathrm{LL}_{3}{ }^{*}$ & $4.89 \pm 1.40$ & $4.91 \pm 1.03$ & $4.90 \pm 1.13$ & $5.55 \pm 0.80$ & $4.51 \pm 0.77$ & $5.34 \pm 0.93^{c}$ \\
\hline $\mathrm{LL}_{4}^{* *}$ & $10.97 \pm 1.47$ & $11.65 \pm 1.37$ & $11.48 \pm 1.2$ & $12.41 \pm 1.07$ & $11.28 \pm 1.39$ & $12.21 \pm 1.62$ \\
\hline $\mathrm{LL}_{5}$ & $17.94 \pm 1.67$ & $18.88 \pm 1.77$ & $17.67 \pm 1.12$ & $18.96 \pm 1.00^{\mathrm{bb}}$ & $17.47 \pm 2.43$ & $19.00 \pm 1.70$ \\
\hline $\mathrm{LLm}_{6}^{* *}$ & $24.97 \pm 1.99$ & $25.63 \pm 1.85$ & $24.45 \pm 1.36$ & $25.78 \pm 1.36^{\mathrm{b}}$ & $24.85 \pm 1.60$ & $26.36 \pm 1.75^{\mathrm{c}}$ \\
\hline $\operatorname{LLd}_{6}^{* *}$ & $29.33 \pm 1.95$ & $30.13 \pm 1.86$ & $28.62 \pm 1.53$ & $30.55 \pm 1.34^{\mathrm{bb}}$ & $29.59 \pm 1.70$ & $30.65 \pm 1.87$ \\
\hline $\mathrm{LLm}_{7}^{* *}$ & $35.16 \pm 2.01$ & $36.69 \pm 1.96^{\mathrm{a}}$ & $34.25 \pm 2.06$ & $36.78 \pm 1.16^{\mathrm{bb}}$ & $35.62 \pm 1.92$ & $37.18 \pm 2.45^{\mathrm{c}}$ \\
\hline $\mathrm{LLd}_{7}$ & $39.17 \pm 2.16$ & $40.60 \pm 1.95$ & $39.00 \pm 1.83$ & $41.31 \pm 1.11^{\mathrm{bb}}$ & $39.72 \pm 1.97$ & $41.32 \pm 2.69$ \\
\hline UAC-C & $39.33 \pm 1.96$ & $39.77 \pm 2.66$ & $38.91 \pm 1.70$ & $40.41 \pm 1.18^{\mathrm{bb}}$ & $37.39 \pm 1.41$ & $38.05 \pm 2.38$ \\
\hline LAC-C & $31.59 \pm 2.63$ & $32.06 \pm 1.58$ & $30.17 \pm 1.50$ & $30.99 \pm 1.35$ & $31.52 \pm 0.93$ & $32.89 \pm 1.78^{c}$ \\
\hline UAP-P** & $49.38 \pm 1.93$ & $51.48 \pm 2.58^{\mathrm{a}}$ & $48.91 \pm 2.14$ & $50.63 \pm 1.91^{\mathrm{b}}$ & $48.69 \pm 1.94$ & $49.40 \pm 2.73$ \\
\hline LAP-P & $42.73 \pm 2.13$ & $44.83 \pm 1.39^{\text {aa }}$ & $42.98 \pm 1.75$ & $44.04 \pm 1.86$ & $43.75 \pm 1.67$ & $45.32 \pm 1.86^{\mathrm{c}}$ \\
\hline UAM-M $^{* *}$ & $58.20 \pm 2.19$ & $61.04 \pm 3.52^{\text {aa }}$ & $57.57 \pm 1.76$ & $59.75 \pm 1.73^{b}$ & $58.41 \pm 1.40$ & $58.69 \pm 2.91$ \\
\hline LAM-M & $54.73 \pm 2.59$ & $56.46 \pm 3.32$ & $54.21 \pm 1.84$ & $56.18 \pm 1.69^{\mathrm{bb}}$ & $56.31 \pm 1.44$ & $56.78 \pm 2.53$ \\
\hline Palate Height ${ }^{* *}$ & $20.02 \pm 1.43$ & $21.18 \pm 1.89$ & $19.81 \pm 1.34$ & $20.70 \pm 1.39$ & $19.83 \pm 1.69$ & $22.29 \pm 2.46^{\mathrm{cc}}$ \\
\hline
\end{tabular}

ACI: Angle Class I malocclusion; ACII: Angle Class II malocclusion; ACIII: Angle Class III malocclusion; ${ }^{\text {a }}<<0.05$ and ${ }^{\text {aa }} \mathrm{p}<0.01$ between genders in ACI; ${ }^{b} p<0.05$ and ${ }^{b b} p<0.01$ between genders in ACII; ${ }^{c} p<0.05$ and ${ }^{c c} p<0.01$ between genders in ACIII;

${ }^{*} \mathrm{p}<0.05$ and $* * \mathrm{p}<0.01$ gender difference; $\& \mathrm{p}<0.05$ and $\& \& \mathrm{p}<0.01$ Group difference; $\# \mathrm{p}<0.05$ and \#\# $\mathrm{p}<0.01$ interaction of group and gender differences. 
Citation: Zhou, N., et al. Evaluation of Lingual Arch form among Angle Class I, Class Iiand Class III Malocclusions in Chinese Orthodontic Patients. (2018) J Dent Oral Care 4(2): 30- 37.

Statistically significant differences of maxillary and mandibular dental arch and alveolar width dimensions were found in LLm ${ }_{7}$, UAP- P, LAP- P and UAM- M between male and female among the ACI group, these measurements of male are greater than those of female, $p<0.05$. In the ACII group, men's dimensions of $\mathrm{UW}_{2}, \mathrm{UW}_{3}, \mathrm{UW}_{4}, \mathrm{UW}_{5}, \mathrm{UWd}_{6}, \mathrm{UWm}_{7}$, $\mathrm{UWd}_{7}, \mathrm{LW}_{3}, \mathrm{LWd}_{6}, \mathrm{LWm}_{7}, \mathrm{LWd}_{7}, \mathrm{UL}_{2}, \mathrm{UL}_{3}, \mathrm{UL}_{4}, \mathrm{UL}_{5}, \mathrm{ULm}_{6}$, $\mathrm{ULd}_{6}, \mathrm{ULm}_{7}, \mathrm{ULd}_{7}, \mathrm{LL}_{2}, \mathrm{LL}_{5}, \mathrm{LLm}_{6}, \mathrm{LLd}_{6}, \mathrm{LLm}_{7}, \mathrm{LLd}_{7}, \mathrm{UAC}-\mathrm{C}$, UAP-P, UAM-M and LAM-M were significantly greater than those of female. In the ACIII group, statistically significant higher dimensions were found in $\mathrm{UWd}_{7}, \mathrm{LWd}_{7}, \mathrm{UL}_{3}, \mathrm{UL}_{4}, \mathrm{UL}_{5}$, $\mathrm{ULm}_{6}, \mathrm{ULd}_{6}, \mathrm{ULm}_{7}, \mathrm{ULd}_{7}, \mathrm{LL}_{2}, \mathrm{LL}_{3}, \mathrm{LLm}_{6}, \mathrm{LLm}_{7}, \mathrm{LAC}-\mathrm{C}$, LAP-P and palate height in male than female, $\mathrm{p}<0.05$.

Group differences on Angle's Class I, II or III malocclusions Descriptive statistics and statistical comparisons of dental lingual arch widths and lengths and alveolar width measurements are shown in Table 4. Statistically significant differences of maxillary and mandibular dental arch and alveolar width dimensions were found $\mathrm{inLW}_{2}, \mathrm{UL}_{1}$ and $\mathrm{LAC}-\mathrm{C}$ between ACII and ACI groups; the significant differences of $\mathrm{LW}_{3}, \mathrm{LWm}_{6}, \mathrm{LWd}_{6}$, $\mathrm{UL}_{1}, \mathrm{UL}_{3}, \mathrm{UL}_{4}, \mathrm{UL}_{5}, \mathrm{ULm}_{6}, \mathrm{ULd}_{6}, \mathrm{ULm}_{7}, \mathrm{ULd}_{7}, \mathrm{LL}_{2}, \mathrm{UAC}-\mathrm{C}$ and UAP-P were found between groups of ACIII and ACI; and between ACII and ACIII these groups differences were significant in $\mathrm{UW}_{1}, \mathrm{UWm}_{6}, \mathrm{UWd}_{7}, \mathrm{UWm}_{7}, \mathrm{LW}_{2}, \mathrm{LW}_{3}, \mathrm{LWm}_{6}, \mathrm{LWd}_{6}$, $\mathrm{LWd}_{7}, \mathrm{UL}_{3}, \mathrm{UL}_{4}, \mathrm{UL}_{5}, \mathrm{ULm}_{6}, \mathrm{ULd}_{6}, \mathrm{ULm}_{7}, \mathrm{ULd}_{7}, \mathrm{LL}_{1}, \mathrm{LL}_{2}$, $\mathrm{LAC}-\mathrm{C}$ and UAC-C .

Table 4: Descriptive statistics of maxillary and mandibular lingual arch widths \& lengths and alveolar width measurements among AC malocclusions.

\begin{tabular}{|l|c|c|c|}
\hline Parameters & ACI $(\mathrm{mm})$ & ACII $(\mathrm{mm})$ & ACIII $(\mathrm{mm})$ \\
\hline $\mathrm{UW}_{1}$ & $8.65 \pm 0.90$ & $8.75 \pm 0.69$ & $8.23 \pm 0.71^{\mathrm{cc}}$ \\
\hline $\mathrm{UW}_{2}$ & $21.31 \pm 1.54$ & $21.22 \pm 1.69$ & $20.79 \pm 1.61$ \\
\hline $\mathrm{UW}_{3}$ & $31.2 \pm 2.25$ & $30.88 \pm 1.79$ & $31.27 \pm 3.29$ \\
\hline $\mathrm{UW}_{4}$ & $30.41 \pm 2.54$ & $29.88 \pm 2.25$ & $30.76 \pm 2.14$ \\
\hline $\mathrm{UW}_{5}$ & $35.36 \pm 3.28$ & $33.85 \pm 2.01$ & $35.17 \pm 2.58$ \\
\hline $\mathrm{UWm}_{6}$ & $38.13 \pm 3.40$ & $36.41 \pm 2.23$ & $38.56 \pm 2.39^{\mathrm{cc}}$ \\
\hline $\mathrm{UWd}_{6}$ & $40.34 \pm 3.57$ & $38.71 \pm 2.59$ & $40.95 \pm 2.63^{\mathrm{c}}$ \\
\hline $\mathrm{UWm}_{7}$ & $43.42 \pm 4.31$ & $41.76 \pm 2.53$ & $44.12 \pm 2.85^{\mathrm{c}}$ \\
\hline $\mathrm{UWd}_{7}$ & $46.37 \pm 4.65$ & $44.21 \pm 2.58$ & $45.8 \pm 2.95$ \\
\hline $\mathrm{LW}_{1}$ & $5.49 \pm 0.31$ & $5.39 \pm 0.39$ & $5.39 \pm 0.34$ \\
\hline $\mathrm{LW}_{2}$ & $15.49 \pm 1.79$ & $14.39 \pm 1.36^{\mathrm{a}}$ & $15.32 \pm 0.78^{\mathrm{c}}$ \\
\hline $\mathrm{LW}_{3}$ & $23.91 \pm 2.12$ & $23.99 \pm 1.74$ & $25.27 \pm 1.64^{\mathrm{b}}, \mathrm{c}$ \\
\hline $\mathrm{LW}_{4}$ & $27.35 \pm 2.89$ & $27.37 \pm 1.62$ & $28.06 \pm 2.09$ \\
\hline $\mathrm{LW}_{5}$ & $31.24 \pm 3.06$ & $30.90 \pm 2.11$ & $32.08 \pm 2.57$ \\
\hline $\mathrm{LWm}_{6}$ & $33.46 \pm 3.34$ & $33.15 \pm 2.09$ & $35.10 \pm 2.4^{\mathrm{b}}{ }^{\mathrm{c}}$ \\
\hline $\mathrm{LWd}_{6}$ & $35.93 \pm 3.42$ & $35.8 \pm 2.18$ & $37.71 \pm 2.83^{\mathrm{b}}$ \\
\hline $\mathrm{LWm}_{7}$ & $39.51 \pm 3.59$ & $39.04 \pm 2.31$ & $40.62 \pm 2.51$ \\
\hline $\mathrm{LWd}_{7}$ & $41.93 \pm 3.97$ & $40.88 \pm 2.27$ & $42.86 \pm 2.79^{\mathrm{c}}$ \\
\hline $\mathrm{UL}_{1}$ & $0.60 \pm 0.11$ & $0.69 \pm 0.07^{\mathrm{aa}}$ & $0.69 \pm 0.08^{\mathrm{bb}}$ \\
\hline $\mathrm{UL}_{2}$ & $3.31 \pm 0.67$ & $3.69 \pm 0.70$ & $3.35 \pm 1.11$ \\
\hline $\mathrm{UL}_{3}$ & $8.53 \pm 1.29$ & $8.86 \pm 1.28$ & $6.81 \pm 1.68^{\mathrm{bb}}{ }^{\mathrm{cc}}$ \\
\hline $\mathrm{UL}_{4}$ & $17.33 \pm 1.58$ & $17.77 \pm 1.37$ & $15.43 \pm 2.22^{\mathrm{bb}}{ }^{\mathrm{cc}}$ \\
\hline
\end{tabular}

\begin{tabular}{|c|c|c|c|}
\hline $\mathrm{UL}_{5}$ & $24.10 \pm 1.85$ & $24.54 \pm 1.90$ & $22.41 \pm 2.34$ bb ${ }^{\mathrm{cc}}$ \\
\hline $\mathrm{ULm}_{6}$ & $31.03 \pm 2.18$ & $31.48 \pm 1.72$ & $29.22 \pm 2.49^{\mathrm{bb}},{ }^{\mathrm{cc}}$ \\
\hline $\mathrm{ULd}_{6}$ & $35.41 \pm 2.29$ & $36.19 \pm 1.82$ & $33.61 \pm 2.53^{\mathrm{bb}}, \mathrm{cc}$ \\
\hline $\mathrm{ULm}_{7}$ & $41.39 \pm 2.55$ & $41.86 \pm 2.12$ & $39.32 \pm 2.51^{\mathrm{bb}},{ }^{\mathrm{cc}}$ \\
\hline $\mathrm{ULd}_{7}$ & $44.87 \pm 2.45$ & $45.53 \pm 2.05$ & $42.91 \pm 2.54{ }^{\mathrm{bb}},{ }^{\mathrm{cc}}$ \\
\hline $\mathrm{LL}_{1}$ & $0.45 \pm 0.09$ & $0.48 \pm 0.07$ & $0.41 \pm 0.05^{\mathrm{cc}}$ \\
\hline $\mathrm{LL}_{2}$ & $1.92 \pm 0.50$ & $1.96 \pm 0.20$ & $1.69 \pm 0.22^{\mathrm{b}},{ }^{\mathrm{cc}}$ \\
\hline $\mathrm{LL}_{3}$ & $4.90 \pm 1.21$ & $5.22 \pm 1.02$ & $4.93 \pm 0.94$ \\
\hline $\mathrm{LL}_{4}$ & $11.31 \pm 1.44$ & $11.95 \pm 1.21$ & $11.74 \pm 1.56$ \\
\hline $\mathrm{LL}_{5}$ & $18.41 \pm 1.76$ & $18.31 \pm 1.23$ & $18.23 \pm 2.21$ \\
\hline $\mathrm{LLm}_{6}$ & $25.30 \pm 1.92$ & $25.11 \pm 1.50$ & $25.61 \pm 1.82$ \\
\hline $\mathrm{LLd}_{6}$ & $29.73 \pm 1.92$ & $29.59 \pm 1.72$ & $30.12 \pm 1.84$ \\
\hline $\mathrm{LLm}_{7}$ & $35.93 \pm 2.10$ & $35.52 \pm 2.09$ & $36.40 \pm 2.30$ \\
\hline $\mathrm{LLd}_{7}$ & $39.89 \pm 2.15$ & $40.16 \pm 1.89$ & $40.52 \pm 2.46$ \\
\hline UAC-C & $39.55 \pm 2.30$ & $39.66 \pm 1.63$ & $37.72 \pm 1.95^{\mathrm{bb}},{ }^{\mathrm{cc}}$ \\
\hline LAC-C & $31.82 \pm 2.15$ & $30.58 \pm 1.46^{\mathrm{a}}$ & $32.20 \pm 1.55^{\mathrm{cc}}$ \\
\hline UAP-P & $50.43 \pm 2.48$ & $49.77 \pm 2.17$ & $49.04 \pm 2.35^{b}$ \\
\hline LAP-P & $43.78 \pm 2.06$ & $43.51 \pm 1.86$ & $44.54 \pm 1.91$ \\
\hline UAM-M & $59.62 \pm 3.23$ & $58.66 \pm 2.04$ & $58.55 \pm 2.25$ \\
\hline LAM-M & $55.60 \pm 3.06$ & $55.19 \pm 2.00$ & $56.55 \pm 2.04$ \\
\hline Palate Height & $20.60 \pm 1.75$ & $20.26 \pm 1.41$ & $21.06 \pm 2.42$ \\
\hline
\end{tabular}

ACI: Angle Class I malocclusion; ACII: Angle Class II malocclusion; ACIII: Angle Class III malocclusion; ${ }^{a}, \mathrm{ACII}$ vs. ACI, $\mathrm{p}<0.05$; ${ }^{\text {aa }}$, ACII vs ACI, $\mathrm{p}<0.01$; $^{\mathrm{b}}$, ACIII vs ACI, $\mathrm{p}<0.05$; ${ }^{\mathrm{b}}$, ACIII vs. ACI, $\mathrm{p}<0.01$; ${ }^{\mathrm{c}}$, ACIII vs. ACII, $\mathrm{p}<0.05$; ${ }^{\mathrm{cc}}$, ACIII vs. ACII, $\mathrm{p}<0.01$.

Female differences on Angle's Class I, II or III malocclusions Descriptive statistics and statistical comparisons of dental lingual arch widths and lengths and alveolar width measurements are shown in Table 5. Significant differences of maxillary and mandibular dental arch and alveolar width dimensions were found in $\mathrm{UW}_{2}, \mathrm{LW}_{2}, \mathrm{UL}_{1}$ and $\mathrm{LL}_{1}$ between groups of ACII and ACI; the significant differences of $\mathrm{LW}_{3}, \mathrm{UL}_{1}, \mathrm{UL}_{3}, \mathrm{UL}_{4}, \mathrm{UL}_{5}$, $\mathrm{ULm}_{6}, \mathrm{ULd}_{6}, \mathrm{ULm}_{7}, \mathrm{ULd}_{7}$ and UAC-C were also found between ACIII and ACI; and between ACII and ACIII groups differences were significant in $\mathrm{UW}_{5}, \mathrm{UWm}_{6}, \mathrm{UWd}_{6}, \mathrm{UWm}_{7}, \mathrm{LW}_{3}, \mathrm{LWm}_{6}$, $\mathrm{LWd}_{6}, \mathrm{LWm}_{7}, \mathrm{LWd}_{7}, \mathrm{UL}_{3}, \mathrm{UL}_{4}, \mathrm{UL}_{5}, \mathrm{ULm}_{6}, \mathrm{ULd}_{6}, \mathrm{ULm}_{7}, \mathrm{ULd}_{7}$, LL1, LL, LAC-C and LAM-M.

Table 5: Female's descriptive statistics of maxillary and mandibular lingual arch widths and lengths and alveolar width measurements among AC malocclusions.

\begin{tabular}{|l|c|c|c|}
\hline Parameters & ACI $(\mathrm{mm})$ & ACII $(\mathrm{mm})$ & ACIII $(\mathrm{mm})$ \\
\hline $\mathrm{UW}_{1}$ & $8.45 \pm 0.58$ & $8.54 \pm 0.77$ & $8.20 \pm 0.58$ \\
\hline $\mathrm{UW}_{2}$ & $21.46 \pm 0.85$ & $20.34 \pm 1.73^{\mathrm{a}}$ & $20.65 \pm 1.28$ \\
\hline $\mathrm{UW}_{3}$ & $31.20 \pm 1.91$ & $30.14 \pm 1.84$ & $31.74 \pm 1.70$ \\
\hline $\mathrm{UW}_{4}$ & $30.01 \pm 2.33$ & $28.88 \pm 2.39$ & $30.44 \pm 2.06$ \\
\hline $\mathrm{UW}_{5}$ & $34.56 \pm 2.70$ & $32.83 \pm 2.09$ & $34.84 \pm 2.53^{\mathrm{c}}$ \\
\hline $\mathrm{UWm}_{6}$ & $36.95 \pm 2.73$ & $35.55 \pm 2.47$ & $38.26 \pm 1.70^{\mathrm{cc}}$ \\
\hline $\mathrm{UWd}_{6}$ & $39.24 \pm 2.83$ & $37.49 \pm 2.55$ & $40.35 \pm 1.81^{\mathrm{c}}$ \\
\hline $\mathrm{UWm}_{7}$ & $41.94 \pm 3.02$ & $40.52 \pm 2.26$ & $43.18 \pm 1.76^{\mathrm{c}}$ \\
\hline $\mathrm{UWd}_{7}$ & $44.96 \pm 3.45$ & $43.28 \pm 2.43$ & $44.67 \pm 2.01$ \\
\hline
\end{tabular}




\begin{tabular}{|c|c|c|c|}
\hline $\mathrm{LW}_{1}$ & $5.51 \pm 0.21$ & $5.27 \pm 0.29$ & $5.4 \pm 0.37$ \\
\hline $\mathrm{LW}_{2}$ & $15.56 \pm 1.92$ & $13.9 \pm 1.58^{\mathrm{a}}$ & $15.37 \pm 0.80$ \\
\hline $\mathrm{LW}_{3}$ & $23.41 \pm 2.31$ & $23.07 \pm 1.62$ & $25.29 \pm 1.32^{\mathrm{b}},{ }^{\mathrm{cc}}$ \\
\hline $\mathrm{LW}_{4}$ & $27.04 \pm 2.63$ & $26.91 \pm 1.81$ & $28.09 \pm 1.78$ \\
\hline $\mathrm{LW}_{5}$ & $31.01 \pm 2.63$ & $30.26 \pm 2.38$ & $31.71 \pm 2.37$ \\
\hline $\mathrm{LWm}_{6}$ & $32.91 \pm 2.83$ & $32.35 \pm 2.18$ & $34.66 \pm 1.79^{c}$ \\
\hline $\mathrm{LWd}_{6}$ & $34.96 \pm 2.76$ & $34.74 \pm 1.91$ & $36.93 \pm 1.95^{\mathrm{c}}$ \\
\hline $\mathrm{LWm}_{7}$ & $38.70 \pm 2.94$ & $37.98 \pm 2.15$ & $39.83 \pm 1.54^{\mathrm{c}}$ \\
\hline $\mathrm{LWd}_{7}$ & $40.43 \pm 2.90$ & $39.76 \pm 1.85$ & $41.78 \pm 1.67^{\mathrm{c}}$ \\
\hline $\mathrm{UL}_{1}$ & $0.57 \pm 0.12$ & $0.68 \pm 0.08$ aa & $0.67 \pm 0.08^{\mathrm{bb}}$ \\
\hline $\mathrm{UL}_{2}$ & $3.06 \pm 0.80$ & $3.23 \pm 0.52$ & $3.15 \pm 0.81$ \\
\hline $\mathrm{UL}_{3}$ & $8.32 \pm 1.36$ & $8.16 \pm 0.77$ & $6.04 \pm 1.02^{\mathrm{bb}},{ }^{\mathrm{cc}}$ \\
\hline $\mathrm{UL}_{4}$ & $16.85 \pm 1.45$ & $17.21 \pm 1.38$ & $14.27 \pm 1.37^{\mathrm{bb}}, \mathrm{cc}$ \\
\hline $\mathrm{UL}_{5}$ & $23.71 \pm 1.61$ & $23.36 \pm 1.53$ & $21.15 \pm 1.457^{\mathrm{bb}},{ }^{\mathrm{cc}}$ \\
\hline $\mathrm{ULm}_{6}$ & $30.67 \pm 2.18$ & $30.37 \pm 1.57$ & $27.67 \pm 1.17^{\mathrm{bb}},{ }^{\mathrm{cc}}$ \\
\hline $\mathrm{ULd}_{6}$ & $34.98 \pm 2.18$ & $34.97 \pm 1.48$ & $32.18 \pm 1.38^{\mathrm{bb}},{ }^{\mathrm{cc}}$ \\
\hline $\mathrm{ULm}_{7}$ & $41.00 \pm 2.30$ & $40.41 \pm 1.90$ & $37.91 \pm 1.40^{\mathrm{bb}},{ }^{\mathrm{cc}}$ \\
\hline $\mathrm{ULd}_{7}$ & $44.22 \pm 2.12$ & $44.26 \pm 2.00$ & $41.57 \pm 1.49^{\mathrm{bb}},{ }^{\mathrm{cc}}$ \\
\hline $\mathrm{LL}_{1}$ & $0.44 \pm 0.10$ & $0.50 \pm 0.06^{\mathrm{a}}$ & $0.39 \pm 0.05^{\mathrm{cc}}$ \\
\hline $\mathrm{LL}_{2}$ & $1.80 \pm 0.64$ & $1.88 \pm 0.21$ & $1.59 \pm 0.19^{\mathrm{cc}}$ \\
\hline $\mathrm{LL}_{3}$ & $4.89 \pm 1.40$ & $4.90 \pm 1.13$ & $4.51 \pm 0.77$ \\
\hline $\mathrm{LL}_{4}$ & $10.97 \pm 1.47$ & $11.48 \pm 1.20$ & $11.28 \pm 1.39$ \\
\hline $\mathrm{LL}_{5}$ & $17.94 \pm 1.67$ & $17.67 \pm 1.12$ & $17.47 \pm 2.43$ \\
\hline $\mathrm{LLm}_{6}$ & $24.97 \pm 1.99$ & $24.45 \pm 1.36$ & $24.85 \pm 1.6$ \\
\hline $\mathrm{LLd}_{6}$ & $29.33 \pm 1.95$ & $28.62 \pm 1.53$ & $29.59 \pm 1.7$ \\
\hline $\mathrm{LLm}_{7}$ & $35.16 \pm 2.01$ & $34.25 \pm 2.06$ & $35.62 \pm 1.92$ \\
\hline $\mathrm{LLd}_{7}$ & $39.17 \pm 2.16$ & $39.00 \pm 1.83$ & $39.72 \pm 1.97$ \\
\hline UAC-C & $39.33 \pm 1.96$ & $38.91 \pm 1.70$ & $37.39 \pm 1.41^{\mathrm{b}}$ \\
\hline LAC-C & $31.59 \pm 2.63$ & $30.17 \pm 1.50$ & $31.52 \pm 0.93^{\mathrm{c}}$ \\
\hline UAP-P & $49.38 \pm 1.93$ & $48.91 \pm 2.14$ & $48.69 \pm 1.94$ \\
\hline LAP-P & $42.73 \pm 2.13$ & $42.98 \pm 1.75$ & $43.75 \pm 1.67$ \\
\hline UAM-M & $58.2 \pm 2.19$ & $57.57 \pm 1.76$ & $58.41 \pm 1.40$ \\
\hline LAM-M & $54.73 \pm 2.59$ & $54.21 \pm 1.84$ & $56.31 \pm 1.44^{\mathrm{c}}$ \\
\hline Palate Height & $20.02 \pm 1.43$ & $19.81 \pm 1.34$ & $19.83 \pm 1.69$ \\
\hline
\end{tabular}

ACI: Angle Class I malocclusion; ACII: Angle Class II malocclusion; ACIII: Angle Class III malocclusion; ${ }^{\text {a }, ~ A C I I ~ v s . ~ A C I, ~} \mathrm{p}<0.05$; ${ }^{\text {aa }}$, ACII vs ACI, $<<0.01$; ${ }^{b}, A C I I I$ vs ACI, $p<0.05$; ${ }^{b b}, A C I I I$ vs. ACI, $p<0.01 ;{ }^{c}$, ACIII vs. ACII, $\mathrm{p}<0.05$; ${ }^{\mathrm{cc}}$, ACIII vs. ACII, $\mathrm{p}<0.01$.

Male differences on Angle's Class I, II or III malocclusions Descriptive statistics and statistical comparisons of dental lingual arch widths and lengths and alveolar width measurements are shown in Table 6. Significant differences of maxillary and mandibular dental arch and alveolar width dimensions were only found in UL1 when ACII compared to ACI, ACII group is greater than ACI group, $\mathrm{p}<0.05$; the significant differences of $\mathrm{UL}_{3}, \mathrm{LL}_{2}$, UAP-P and UAM-M were also found between ACIII and ACI, ACIII group is smaller than ACI group, $\mathrm{p}<0.05$; and when ACII compared to ACIII group differences were significant in $\mathrm{UW}_{1}$, $\mathrm{UW}_{2}, \mathrm{UL}_{3}, \mathrm{UL}_{4}, \mathrm{UL}_{5}, \mathrm{ULm}_{6}, \mathrm{ULd}_{6}, \mathrm{ULm}_{7}, \mathrm{ULd}_{7}, \mathrm{LL}_{2}, \mathrm{UAC}-\mathrm{C}$, LAC-C and palate height.

Table 6: Males descriptive statistics of maxillary and mandibular lingual arch widths and lengths and alveolar width measurements among AC malocclusions.

\begin{tabular}{|c|c|c|c|}
\hline Parameters & $\mathrm{ACI}(\mathrm{mm})$ & $\mathrm{ACII}(\mathrm{mm})$ & $\mathrm{ACIII}(\mathrm{mm})$ \\
\hline $\mathrm{UW}_{1}$ & $8.85 \pm 1.13$ & $8.95 \pm 0.57$ & $8.25 \pm 0.84^{c}$ \\
\hline $\mathrm{UW}_{2}$ & $21.17 \pm 2.04$ & $22.10 \pm 1.13$ & $20.93 \pm 1.06^{c}$ \\
\hline $\mathrm{UW}_{3}$ & $31.19 \pm 2.61$ & $31.63 \pm 1.45$ & $30.81 \pm 4.37$ \\
\hline $\mathrm{UW}_{4}$ & $30.81 \pm 2.76$ & $30.89 \pm 1.63$ & $31.07 \pm 2.23$ \\
\hline $\mathrm{UW}_{5}$ & $36.16 \pm 3.96$ & $34.88 \pm 1.31$ & $35.49 \pm 2.67$ \\
\hline $\mathrm{UWm}_{6}$ & $39.31 \pm 3.67$ & $37.27 \pm 1.63$ & $38.86 \pm 2.96$ \\
\hline $\mathrm{UWd}_{6}$ & $41.43 \pm 3.97$ & $39.94 \pm 2.03$ & $41.55 \pm 3.21$ \\
\hline $\mathrm{UWm}_{7}$ & $44.89 \pm 4.97$ & $43.01 \pm 2.19$ & $45.05 \pm 3.45$ \\
\hline $\mathrm{UWd}_{7}$ & $47.78 \pm 5.35$ & $45.15 \pm 2.44$ & $46.93 \pm 3.35$ \\
\hline $\mathrm{LW}_{1}$ & $5.47 \pm 0.40$ & $5.50 \pm 0.45$ & $5.39 \pm 0.33$ \\
\hline $\mathrm{LW}_{2}$ & $15.42 \pm 1.71$ & $14.88 \pm 0.92$ & $15.26 \pm 0.77$ \\
\hline $\mathrm{LW}_{3}$ & $24.41 \pm 1.86$ & $24.91 \pm 1.36$ & $25.25 \pm 1.97$ \\
\hline $\mathrm{LW}_{4}$ & $27.67 \pm 3.20$ & $27.84 \pm 1.30$ & $28.03 \pm 2.42$ \\
\hline $\mathrm{LW}_{5}$ & $31.46 \pm 3.51$ & $31.55 \pm 1.63$ & $32.46 \pm 2.79$ \\
\hline $\mathrm{LWm}_{6}$ & $34.02 \pm 3.8$ & $33.95 \pm 1.72$ & $35.53 \pm 2.89$ \\
\hline $\mathrm{LWd}_{6}$ & $36.90 \pm 3.81$ & $36.86 \pm 1.96$ & $38.49 \pm 3.38$ \\
\hline $\mathrm{LWm}_{7}$ & $40.30 \pm 4.01$ & $40.10 \pm 2.00$ & $41.4 \pm 3.06$ \\
\hline $\mathrm{LWd}_{7}$ & $42.53 \pm 3.85$ & $42.00 \pm 2.14$ & $43.93 \pm 3.30$ \\
\hline $\mathrm{UL}_{1}$ & $0.64 \pm 0.09$ & $0.70 \pm 0.07^{\mathrm{a}}$ & $0.70 \pm 0.09$ \\
\hline $\mathrm{UL}_{2}$ & $3.56 \pm 0.37$ & $4.15 \pm 0.55$ & $3.56 \pm 1.33$ \\
\hline $\mathrm{UL}_{3}$ & $8.73 \pm 1.23$ & $9.56 \pm 1.33$ & $7.58 \pm 1.88^{\mathrm{b}}, \mathrm{cc}$ \\
\hline $\mathrm{UL}_{4}$ & $17.81 \pm 1.61$ & $18.33 \pm 1.15$ & $16.58 \pm 2.34^{c}$ \\
\hline $\mathrm{UL}_{5}$ & $24.49 \pm 2.05$ & $25.72 \pm 1.46$ & $23.68 \pm 2.4^{\mathrm{cc}}$ \\
\hline $\mathrm{ULm}_{6}$ & $31.38 \pm 2.21$ & $32.59 \pm 1.01$ & $30.77 \pm 2.53 \mathrm{cc}$ \\
\hline $\mathrm{ULd}_{6}$ & $35.84 \pm 2.40$ & $37.41 \pm 1.21$ & $35.03 \pm 2.65^{\mathrm{cc}}$ \\
\hline $\mathrm{ULm}_{7}$ & $41.78 \pm 2.81$ & $43.31 \pm 1.09$ & $40.73 \pm 2.62 \mathrm{cc}$ \\
\hline $\mathrm{ULd}_{7}$ & $45.52 \pm 2.64$ & $46.80 \pm 1.12$ & $44.26 \pm 2.68^{\mathrm{cc}}$ \\
\hline $\mathrm{LL}_{1}$ & $0.46 \pm 0.09$ & $0.46 \pm 0.08$ & $0.43 \pm 0.06$ \\
\hline $\mathrm{LL}_{2}$ & $2.04 \pm 0.25$ & $2.14 \pm 0.16$ & $1.79 \pm 0.20^{\mathrm{b}},{ }^{\mathrm{cc}}$ \\
\hline $\mathrm{LL}_{3}$ & $4.91 \pm 1.03$ & $5.55 \pm 0.80$ & $5.34 \pm 0.93$ \\
\hline $\mathrm{LL}_{4}$ & $11.65 \pm 1.37$ & $12.41 \pm 1.07$ & $12.21 \pm 1.62$ \\
\hline $\mathrm{LL}_{5}$ & $18.88 \pm 1.77$ & $18.96 \pm 1.00$ & $19.00 \pm 1.70$ \\
\hline $\mathrm{LLm}_{6}$ & $25.63 \pm 1.85$ & $25.78 \pm 1.36$ & $26.36 \pm 1.75$ \\
\hline $\operatorname{LLd}_{6}$ & $30.13 \pm 1.86$ & $30.55 \pm 1.34$ & $30.65 \pm 1.87$ \\
\hline $\mathrm{LLm}_{7}$ & $36.69 \pm 1.96$ & $36.78 \pm 1.16$ & $37.18 \pm 2.45$ \\
\hline $\operatorname{LLd}_{7}$ & $40.60 \pm 1.95$ & $41.31 \pm 1.11$ & $41.32 \pm 2.69$ \\
\hline UAC-C & $39.77 \pm 2.66$ & $40.41 \pm 1.18$ & $38.05 \pm 2.38^{\mathrm{cc}}$ \\
\hline LAC-C & $32.06 \pm 1.58$ & $30.99 \pm 1.35$ & $32.89 \pm 1.78^{\mathrm{cc}}$ \\
\hline UAP-P & $51.48 \pm 2.58$ & $50.63 \pm 1.91$ & $49.4 \pm 2.73^{b}$ \\
\hline LAP-P & $44.83 \pm 1.39$ & $44.04 \pm 1.86$ & $45.32 \pm 1.86$ \\
\hline UAM-M & $61.04 \pm 3.52$ & $59.75 \pm 1.73$ & $58.69 \pm 2.91^{\mathrm{bb}}$ \\
\hline LAM-M & $56.46 \pm 3.32$ & $56.18 \pm 1.69$ & $56.78 \pm 2.53$ \\
\hline Palate Height & $21.18 \pm 1.89$ & $20.70 \pm 1.39$ & $22.29 \pm 2.46^{c}$ \\
\hline
\end{tabular}

ACI: Angle Class I malocclusion; ACII: Angle Class II malocclusion; ACIII: Angle Class III malocclusion; a, ACII vs. ACI, $<<0.05$; aa, $\mathrm{ACII}$ vs ACI, $\mathrm{p}<0.01$; ${ }^{\mathrm{b}}$, ACIII vs ACI, $\mathrm{p}<0.05$; ${ }^{\text {bb }}$, ACIII vs. ACI, $\mathrm{p}<0.01$; ${ }^{\mathrm{c}}$, 
Citation: Zhou, N., et al. Evaluation of Lingual Arch form among Angle Class I, Class Iiand Class III Malocclusions in Chinese Orthodontic Patients. (2018) J Dent Oral Care 4(2): 30- 37.

ACIII vs. ACII, $\mathrm{p}<0.05 ;{ }^{\mathrm{cc}}, \mathrm{ACIII}$ vs. ACII, $\mathrm{p}<0.01$.

\section{Discussion}

Over the half century, despite improvements to the lingual orthodontic protocol, more and more Chinese patients experience discomfort and dysfunction ${ }^{[4,14]}$. These problems could be minimized by selecting the most appropriate arch form. A survey of arch size could help the clinician in choosing correctly shaped stock impression trays for prosthodontic treatment. In addition to the selection of stock trays, the sizes of artificial teeth and the overall form of the artificial dental arch at the wax trial stage are amenable to modification by the dental surgeon in orthodontic treatment ${ }^{[15]}$.

This study was carried out to compare the dental arch and alveolar base widths of Angle's Class I, II and III malocclusions. Width and length measurements described in this article will help clinicians diagnose and plan the treatment of patients with these malocclusions. Investigators who have studied transverse arch changes in subjects have reported molar and canine arch widths did not change after 13 years of age in females and 16 years of age in males ${ }^{[16]}$. The minimum ages of the subjects measured in this study were chosen based on these previous studies. We assumed the arch widths of the subjects studied were fully developed.

Clinicians have speculated nasal obstruction, finger habits, tongue thrusting, low tongue position, and abnormal swallowing and sucking behavior were reasons for narrower maxillary dental arch widths in malocclusion patients compared with normal occlusion sample. Invigatinghe form of the human dental arch using 40 sets of pretreatment orthodontic models of patients, researchers found AC III maxillary dental arch widths were $5.1 \mathrm{~mm}$ greater than the arch widths of AC I widths and this begins in the lateral incisor-canine area and proceeds distally ${ }^{[17]}$. This surprising result was explained by frequently referring to the antero posterior skeletal discrepancy and the fact the mandibular arch is advanced relative to the maxillary arch. In contrast, the maxillary interpremolar/intermolar lengths and all maxillary alveolar width measurements were found to be significantly narrower in the ACIII than in the ACI sample. When the corresponding interarch widths and lengths were matched correctly, the maxillary arch widths and lengths were usually narrower than the mandible arch widths. These were in line with some previous studies, which found the overgrowth of the mandible is the main cause of Class III malocclusionin Asian patients $^{[18]}$. It was reported more than $50 \%$ of white American patients with either normal or prognathic mandibles had a deficiency in the maxilla ${ }^{[19]}$.

Gender dimorphism influenced lingual arch width and length regardless of the front, middle and rear sections are similar within ACI malocclusion. Male anterior mandibular molars and maxillary molar alveolar width groove width were larger than females; however, there was no canine alveolar width difference between genders. In AC II lingual arch width, maxillary width and was the width of the mandibular lingual arch anterior and posterior segments were larger in male than female; maxillary and mandibular lingual arch lengths of male were larger than female as well. Men jaw mandibular canine and premolar alveolar width and palate height were larger than females, indi- cating AC III jaw overgrown in men. When the mandibular tooth size is increased, mandibular arch length and arch width increase occurs.

The comparisons of measurement from three groups found there was a significant difference between genders on the maxillary and mandibular middle and posterior segment lengths and maxillary alveolar width of premolars and molars, and palate height. Disagreement among other studies may be explained by several factors, gender dimorphism, ethnic and racial differences, sample selection and size, and age of subjects.

Mandibular lingual intercanine widths and widths of maxillary and mandibular posterior segment, III $>$ II; length of lingual maxillary canine and posterior segment, III $<$ I and II; alveolar widths of mandibular canine and molar, III $>$ II; however, among the three groups of male, lingual length of maxillary canine and posterior segment and the alveolar width of maxillary canine, III < II; Maxillary alveolar widths of premolar and molar, III $<$ I; Palate height of class III malocclusion is greater than class II malocclusion. Combined, mandibular canine alveolar width, II < I and III; maxillary canine alveolar width showed no difference in II and III. These results were distinguishable from what reported previously that AC II subjects with normal occlusion had larger maxillary intercanine widths than the malocclusion subjects, but no differences were found in mandibular intercanine widths ${ }^{[20]}$.

Three types of lingual arch malocclusion of mandibular canine and molar area width are different, AC III malocclusion is greater than AC II, indicating AC III wide arch lateral overgrown, while narrow arch and mandibular deficiency in AC II. Incisor lingual maxillary arch length of AC II was greater than AC I, which may be associated with AC II maxillary incisors or upper lip to a larger tilt, whereas which was shorter in AC I when compared to ACIII, it is possible due to maxillary incisor inclination related compensatory lip. A possible explanation for the increased arch width associated with AC III dental arches is the sum of all the mesiodistal widths of the dental units around an arch represents a specific dimension.

Lingually positioned maxillary posterior cross bites are often seen in the AC III. One could speculate during eruption in AC III subjects, the maxillary posterior teeth compensate for the buccal relationships (result from the anteroposterior displacement of the jaws) by palate movement to avoid inappropriate contacts with the lower teeth. Besides, it was believed a wide and big mandible obstructed growth and development of the maxillary dental and alveolar arches. It had been reported before the mandibular dental arch widths associated with AC III were on $2.1 \mathrm{~mm}$ wider than the AC I mandibular arches beginning in the premolar area ${ }^{[17]}$. The samples in our study were mainly from southern China, which should count into consideration of the differences of regions and racial standards.

Acknowledgement: There are no known conflicts of interest associated with this publication and there has been no significant financial support for this work that could have influenced its outcome.

Conflict of Interest Statement: We confirm that there are no known conflicts of interest associated with this article and there has been no significant financial support for this work that could 
have influenced its outcome.

\section{References}

1. Lee, R.T. Arch width and form: a review. (1999) Am J Orthod Dentofacial Orthop 115(3): 305-313.

Pubmed |Crossref | Others

2. Park, K.H., Bayome, M., Park, J.H., Lee, J.W., et al. New classification of lingual arch form in normal occlusion using three dimensional virtual models. (2015) Korean J Orthod, 45(2): 74-81.

Pubmed |Crossref | Others

3. Handelman, C.S. The anterior alveolus: its importance in limiting orthodontic treatment and its influence on the occurrence of iatrogenic sequelae.(1996) Angle Orthod 66(2): 95-109.

Pubmed | Crossref | Others

4. Fujita, K. New orthodontic treatment with lingual bracket mushroom arch wire appliance. (1979) Am J Orthod, 76(6): 657-675.

Pubmed | Crossref | Others

5. Lombardo, L., Saba, L., Scuzzo, G., et al. A new concept of anatomic lingual arch form. (2010) Am J Orthod Dentofacial Orthop 138(3): 260.

Pubmed | Crossref | Others

6. Kyung, H.M., Park, H.S., Sung, J.H., et al. The Lingual Plain-Wire System with Micro-Implant ancorage. (2004) J Clin Orthod 38(7): 388-395.

Pubmed | Crossref | Others

7. Sayin, M.O., Turkkahraman, H. Comparison of dental arch and alveolar widths of patients with Class II, division 1 malocclusion and subjects with Class I ideal occlusion. (2004) Angle Orthod 74(3): 356-360.

Pubmed | Crossref | Others

8. Kook, Y.A.,, Nojima, K., Moon, H.B., et al. Comparison of arch forms between Korean and North American white populations. (2004) Am J Orthod Dento facial Orthop 126(6): 680-686.

Pubmed |Crossref | Others

9. Oda, S., Arai, K., Nakahara, R. Commercially available archwire forms compared with normal dental arch forms in a Japanese population. (2010) Am J Orthod Dentofacial Orthop 137(4): 520-527.

Pubmed | Crossref | Others

10. Burris, B.G., Harris, E.F. Maxillary arch size and shape in american blacks and whites. (2000) Angle Orthod 70(4): 297-302.

Pubmed | Crossref | Others
11. Basaran, G., Hamamci, N., Hamamci, O. Comparision of dental arch widths in different types of malocclusions. (2008) World J Orthod 9(1): 20-28

Pubmed $\mid$ Crossref $\mid$ Others

12. Uysal, T., Usumez, S., Memili, B., et al. Dental and alveolar arch widths in normal occlusion and Class III malocclusion. (2005) Angle Orthod, 75(5): 809-813.

Pubmed | Crossref | Others

13. Huth, J., Staley, R.N., Jacobs, R., et al. Arch widths in class II-2 adults compared to adults with class II-1 and normal occlusion.(2007) Angle Orthod 77(5): 837-844.

Pubmed |Crossref| Others

14. Schubert, K., Halbich, T., Jost-Brinkmann, P.G., et al. Precision of indirect bonding of lingual brackets using the Quick Modul System (QMS). (2013) J Orofac Orthop 74(1): 6-17. Pubmed | Crossref | Others

15. Kuntz, T.R., Staley, R.N., Bigelow, H.F., et al. Arch widths in adults with Class I crowded and Class III malocclusions compared with normal occlusions. (2008) Angle Orthod, 78(4): 597-603.

Pubmed |Crossref | Others

16. Bishara, S.E., Jakoben, J.R., Treder, J., et al. Arch length changes from 6 weeks to 45 years. (1998) Angle Orthod 68(1): 69-74.

Pubmed |Crossref | Others

17. Braun, S., Hnat, W., Pender, D.E., et al. The form of the human dental arch. (1998) Angle Orthod 68(1): 29-36. Pubmed |Crossref | Others

18. Lee, S.J., Kim, T.W., Suhr, C.H. Study of recognition of malocclusion and orthodontic treatments. (1994) Korean J Orthod 24(1): 367-394.

Pubmed |Crossref | Others

19. Guyer, E.C., Ellis, E.E., McNamara, J.A., et al. Components of Class III malocclusion in juveniles and adolescents. (1986) Angle Orthod, 56(1): 7-30.

Pubmed | Crossref| Others

20. Staley, R.N., Stuntz, W.R., Peterson, L.C. A comparison of arch widths in adults with normal occlusion and adults with class II, Division 1 malocclusion. (1985) Am J Orthod 88(2): 163-169.

Pubmed |Crossref | Others

Submit your manuscript to Ommega Publishers and we will help you at every step:

- We accept pre-submission inquiries

- Our selector tool helps you to find the most relevant journal

- We provide round the clock customer support

- Convenient online submission

- Thorough peer review

- Inclusion in all major indexing services

- Maximum visibility for your research

Submit your manuscript at

OMMEgA Publishers

https://www.ommegaonline.org/submit-manuscript 